\title{
A systematic review and meta-analysis of acupoint application combined with western medicine therapy in the treatment of bronchial asthma
}

\author{
Jun $\mathrm{Hu}^{1,2 \#}$, Cong Zhang ${ }^{1,2 \#}$, Shumei Zhao ${ }^{1,2}$, Kaiwen Ge ${ }^{1,2}$, Kuan $\mathrm{Di}^{1,2}$, Hesheng Wang ${ }^{1,2}$, Lanying Liu ${ }^{1,2}$ \\ ${ }^{1}$ Department of Acupuncture and Rehabilitation, Affiliated Hospital of Nanjing University of Chinese Medicine/Jiangsu Province Hospital of \\ Chinese Medicine, Nanjing, China; ${ }^{2}$ The First Clinical Medical College, Nanjing University of Chinese Medicine, Nanjing, China \\ Contributions: (I) Conception and design: L Liu; (II) Administrative support: H Wang; (III) Provision of study materials or patients: S Zhao, K Ge, \\ K Di; (IV) Collection and assembly of data: C Zhang; (V) Data analysis and interpretation: J Hu; (VI) Manuscript writing: All authors; (VII) Final \\ approval of manuscript: All authors. \\ \#These authors contributed equally to this work. \\ Correspondence to: Hesheng Wang; Lanying Liu. Department of Acupuncture and Rehabilitation, Affiliated Hospital of Nanjing University of Chinese \\ Medicine/Jiangsu Province Hospital of Chinese Medicine, Nanjing 210029, China. Email: wanghesheng2010@126.com; bluelany@163.com.
}

\begin{abstract}
Background: This study aimed to evaluate and compare the efficacy and safety of acupoint application therapy (AAT) with conventional western medicine therapy (CWMT) and CWMT in the treatment of bronchial asthma. Since there are several researches reporting AAT with CWMT for bronchial asthma and there is little comprehensive analysis on this topic, we conducted this research.

Methods: Randomized controlled trials on the use of AAT with CWMT in the treatment of bronchial asthma published between 2009 and 2020 were retrieved from the PubMed, Embase, Cochrane Library, and CNKI (Chinese National Knowledge Institute) databases. Studies meeting the inclusion criteria were selected for meta-analysis. Forest plot, sensitivity analysis and publication bias assessment were carried out in this article.
\end{abstract}

Results: Eight studies involving 1,520 patients were included in the meta-analysis. The clinical effect of AAT with CWMT in the treatment of asthma was superior to that of CWMT [mean difference $(\mathrm{MD})=2.66$ with $95 \%$ confidential interval (CI) $(2.03,3.49)$; overall effect $\mathrm{P}$ value $<0.00001$ and $\left.\mathrm{I}^{2}=89 \%\right]$. There was no difference in adverse events between AAT with CWMT and CWMT [odds ratio (OR) =1.45; 95\% CI: 0.62, 3.39; $\mathrm{I}^{2}=0 \%$ and $\mathrm{P}$ of overall effect $\left.=0.4\right]$. CWMT had higher ineffectiveness rate than AAT with CWMT $\left(\mathrm{OR}=0.29 ; 95 \% \mathrm{CI}: 0.22,0.38 ; \mathrm{P}=0.33 ; \mathrm{I}^{2}=13 \%\right)$. According to the statistical analysis results, the AAT with CWMT group had higher overall effectiveness rate than the CWMT group (OR $=0.29 ; 95 \%$ CI: 0.22, 0.38; $\mathrm{P}=0.33$, fixed-effects model), with low heterogeneity $\left(\mathrm{P}=0.29 ; \mathrm{I}^{2}=13 \%\right)$.

Discussion: AAT with CWMT has a superior clinical effect to CWMT in patients with asthma, and there is no difference in adverse events between the two treatments. Therefore, AAT with CWMT should be promoted as a treatment for bronchial asthma.

Keywords: Acupoint application therapy (AAT); bronchial asthma; western medicine; meta-analysis

Submitted Aug 11, 2021. Accepted for publication Oct 19, 2021.

doi: $10.21037 /$ apm-21-2507

View this article at: https://dx.doi.org/10.21037/apm-21-2507

\section{Introduction}

Western medicine is currently the primary mode of clinical treatment for bronchial asthma. However, the treatment period for bronchial asthma is long $(1,2)$, and long-term use of western medicine is related to drug resistance and adverse reactions $(3,4)$. Although there is improvements 
in the use of conventional western medicines to treat lung function disorders, this approach still lacks efficacy. Therefore, the discovery of more safe, reliable, and longterm bronchial asthma treatment modalities is essential. Conventional western medicine therapy (CWMT) is one of the most effective methods for treating asthma and can improve patients' lung function. Glucocorticoids can serve a therapeutic purpose in several ways (5). Hormone therapy can have significant side effects and is mainly applied, starting with small doses, in the treatment of acute asthma. The selection of medication, application of drugs dose, duration of application, and interval between each application are all based on their constitution and tolerance $(6,7)$. Some hospitals are not equipped to accommodate changes in medication, so patients need to go to a different hospital for care, which increases their time and financial burden. Further, although patients receiving CWMT require hospitalization only once every 4 weeks, they may be infused for 7 days at a time $(8,9)$.

CWMT has the advantages of being low cost and simple (take drugs) compared to AAT with CWMT $(10,11)$. However, as well as having poor overall efficacy, long-term administration of western medicines can cause adverse reactions and the development of drug resistance during treatment (11).

In traditional Chinese medicine (TCM), the treatment should focus on nourishing. Acupoint application therapy (AAT) is a typical TCM. This therapy sticks the medicine to the acupuncture points so that the body can absorb it through the skin. According to the theory of TCM, longterm asthma is related to the internal growth of phlegm and weakness of the spleen and stomach, which results in susceptibility to external evil and induces asthma $(12,13)$. Our previous study demonstrated that Repeated Herbal Acupoint Sticking (RHAS) which is another name for AAT could improve systemic immune response by elevating the Th1/Th2 cell ratio and decreasing the levels of IgE and IL-4, thereby effectively preventing the recurrence of allergic asthma. This study proved the efficacy of a convenient, effective, and hormone independent method for allergic asthma treatment and enhance our understanding of the specific mechanism of TCM treatment of allergic asthma (14).

By searching online databases for studies on the treatment of bronchial asthma, we retrieved a number of published studies involving AAT with CWMT and CWMT. However, there are few meta-analyses comparing the clinical effectiveness and safety of AAT with CWMT with that of CWMT. Therefore, we conducted the present metaanalysis. Our research is an update for AAT with CWMT for the treatment of bronchial asthma with recent published RCTs. We present the following article in accordance with the PRISMA reporting checklist (available at https://dx.doi.org/10.21037/apm-21-2507).

\section{Methods}

\section{Literature search strategy}

We conducted a comprehensive electronic search for randomized controlled trials (RCT) of AAT with CWMT and CWMT in the treatment of bronchial asthma in the PubMed, Embase, Cochrane Library, and CNKI (Chinese National Knowledge Institute) databases. The search was limited to studies published between January 1991 and September 2020. We used the following keywords: "acupoint", "bronchial asthma", "Western medical treatment", and "Chinese herbal medicine". The terms were searched as text words.

\section{Study selection}

The PICOS (participants, interventions, comparators, outcomes, and study design) model was adopted to identify relevant studies for inclusion in this systematic review and meta-analysis. The inclusion criteria for articles were: (I) RCT published in peer-reviewed journals and (II) reported outcome measures relating to the use of AAT with CWMT in the treatment of bronchial asthma. The exclusion criteria were: (I) studies with incomplete text; (II) non-original studies; (III) repetitive research; (IV) reviews, conference and meeting abstracts and presentations, letters, case reports, commentaries, and editorials; (V) studies focusing on treatments other than AAT with CWMT for bronchial asthma; (VI) studies containing only brief descriptions and no comparisons; (VII) studies containing no critical information; (VIII) studies not related to bronchial asthma.

\section{Data extraction and quality assessment}

The titles and abstracts of all publications identified through the search were screened independently ( $\mathrm{Hu}$ and Zhang). 


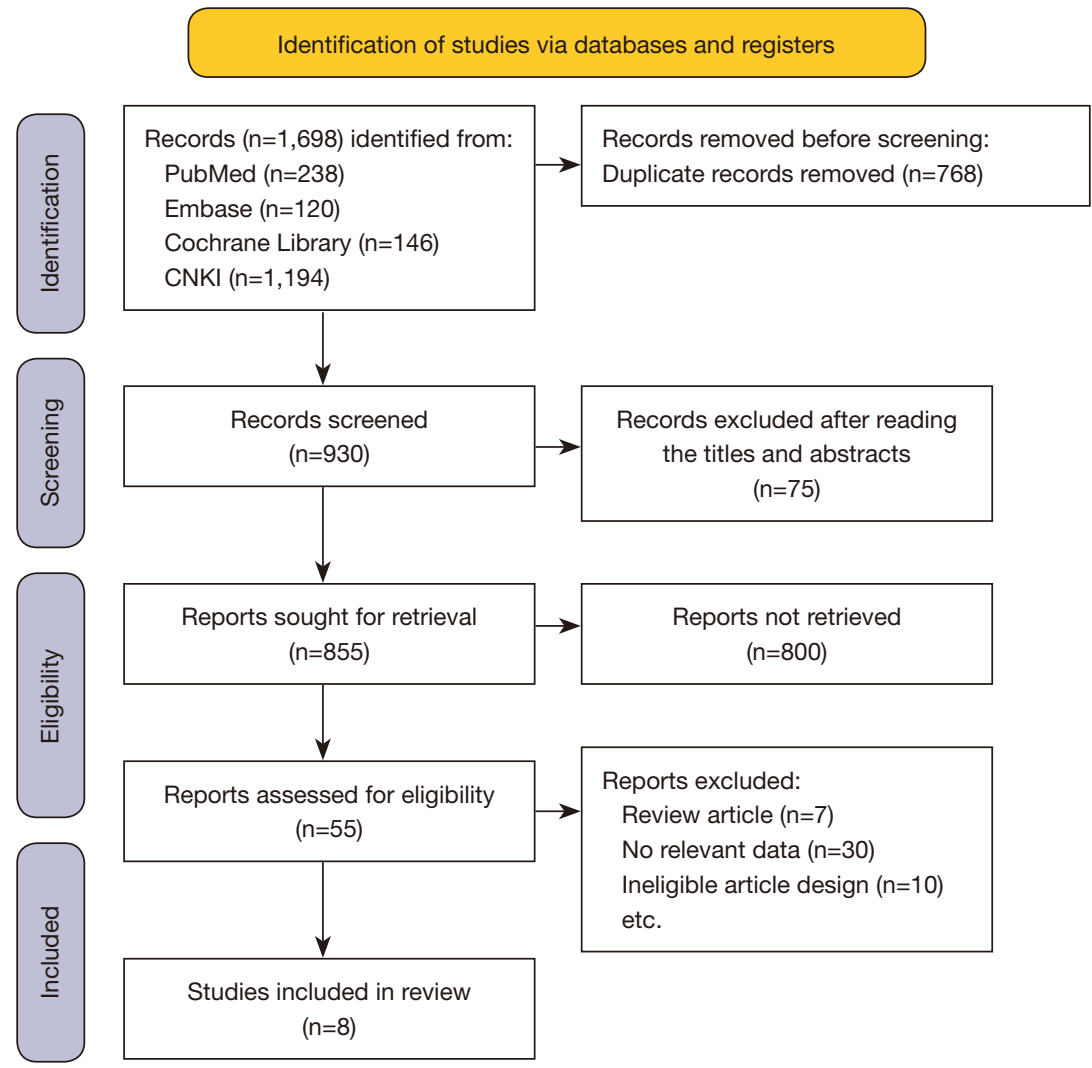

Figure 1 Flow diagram of the study selection process.

Prespecified data, including baseline characteristics, sample size, and the interventions used, were extracted from each trial using a structured data summary table. The Cochrane risk-of-bias tool for randomized controlled trials was used to identify potential bias in the literature.

\section{Statistical analysis}

Statistical analyses were performed using RevMan 5.2 (The Nordic Cochrane Centre, Copenhagen, Denmark). Forest plot in Review Manager Software was used to perform heterogeneity test and sensitivity analysis was performed through deleting one included article in turn to assess the changes in I2 values. Since all outcomes were binary variables and followed a binomial distribution, odds ratios (OR) were used to pool effect sizes. A $\chi^{2}$-based heterogeneity test was performed, and the inconsistency index $\left(\mathrm{I}^{2}\right)$ statistic was determined. Where possible, to explain heterogeneity between trials, we explored a priori hypotheses, which were identified through discussions between our research team and a panel of technical experts before analysis.

\section{Results}

\section{Search process}

A total of 1,698 articles were initially found in the search. We found 930 study titles, but after careful reading of the abstracts and full texts, we concluded that 55 studies met the primary inclusion criteria. After title and abstract review, 47 studies were excluded due to article design, insufficient data and review. Finally, 8 Chinese papers (15-22) were selected for meta-analysis. Detailed information on the search process and the inclusion and exclusion criteria is presented in Figure 1.

\section{Characteristics of the included studies}

The detailed characteristics of the eight eligible studies are summarized in Table 1 . These studies involved 1,520 patients and were all published between 2009 and 2020. The sample 
Table 1 Characteristics of the included studies

\begin{tabular}{|c|c|c|c|c|c|c|c|}
\hline Study & Year & Type of study & Country & Intervention & Sample size (n) & Mean age (years) & Study period \\
\hline Cui (19) & 2009 & $\mathrm{RCT}$ & China & CWMT & 200 & $43 \pm 22.59$ & December 2004 to November 2007 \\
\hline \multirow[t]{2}{*}{ Li et al. (16) } & \multirow[t]{2}{*}{2014} & \multirow[t]{2}{*}{$\mathrm{RCT}$} & \multirow[t]{2}{*}{ China } & AAT with CWMT & 62 & $65.2 \pm 2.37$ & \multirow[t]{2}{*}{ December 2000 to November 2013} \\
\hline & & & & CWMT & 58 & $65.5 \pm 2.71$ & \\
\hline Liu (17) & 2020 & $\mathrm{RCT}$ & China & CWMT & 60 & $8 \pm 2$ & December 2017 to November 2019 \\
\hline \multirow{2}{*}{$\begin{array}{l}\text { Shi et al. } \\
(20)\end{array}$} & \multirow[t]{2}{*}{2011} & \multirow[t]{2}{*}{ RCT } & \multirow[t]{2}{*}{ China } & AAT with CWMT & 40 & $7.9 \pm 1.5$ & \multirow[t]{2}{*}{ December 1998 to November 2008} \\
\hline & & & & CWMT & 40 & $8.2 \pm 1.9$ & \\
\hline $\begin{array}{l}\text { Xuan et al. } \\
\text { (15) }\end{array}$ & 2012 & $\mathrm{RCT}$ & China & AAT with CWMT & 121 & $51.67 \pm 3.72$ & December 2008 to November 2010 \\
\hline Ye et al. (21) & 2017 & RCT & China & CWMT & 50 & $1.89 \pm 0.78$ & December 2000 to November 2004 \\
\hline \multirow{2}{*}{$\begin{array}{l}\text { Yuan et al. } \\
\text { (22) }\end{array}$} & \multirow[t]{2}{*}{2010} & \multirow[t]{2}{*}{$\mathrm{RCT}$} & \multirow[t]{2}{*}{ China } & AAT with CWMT & 51 & $9 \pm 4.76$ & \multirow[t]{2}{*}{ December 2005 to November 2009} \\
\hline & & & & CWMT & 49 & $9 \pm 4.76$ & \\
\hline \multirow{2}{*}{$\begin{array}{l}\text { Zang et al. } \\
\text { (18) }\end{array}$} & \multirow[t]{2}{*}{2009} & $\mathrm{RCT}$ & China & AAT with CWMT & 32 & $43.5 \pm 21.5$ & December 1991 to November 2004 \\
\hline & & & & CWMT & 30 & $46 \pm 18$ & \\
\hline
\end{tabular}

AAT with CWMT, acupoint application; CWMT, conventional western medicine therapy; RCTs, randomized control trials.

sizes ranged from 62 to 700 cases.

\section{Results of quality assessment}

The methodological quality of the included literature was assessed using the Cochrane risk-of-bias tool. Of the eight included articles, two different studies were high risk for performance bias, attrition bias, and reporting bias (Figure 2). A summary of all types of bias for each study is shown in Figure 3.

\section{Results of heterogeneity testing}

An analysis of heterogeneity was conducted to explore differences in clinical effect between AAT with CWMT and CWMT, and the OR was calculated through a metaanalysis based on a fixed-effects model. The overall OR was 2.01 , with $95 \% \mathrm{CI}(1.52,2.66)$. As indicated by the overall effect $\mathrm{P}<0.00001$ and $\mathrm{I}^{2}=36 \%$, the difference between AAT with CWMT and CWMT was significant, with AAT with CWMT having a superior clinical effect to CWMT in the treatment of bronchial asthma (Figure 4).
Heterogeneity analysis of adverse events revealed no difference between AAT with CWMT and CWMT. Metaanalysis based on a fixed-effects model was used to calculate the average difference $(\mathrm{OR}=1.45$; 95\% CI: 0.62, 3.39; $\mathrm{I}^{2}=0 \%$ and $\mathrm{P}$ of overall effect $=0.4$ ) (Figure 5$)$.

Heterogeneity analysis of treatment ineffectiveness revealed a difference between AAT with CWMT and CWMT, and a meta-analysis was subsequently conducted. The results showed a difference in treatment ineffectiveness between AAT with CWMT and CWMT (OR =0.29; 95\% CI: 0.22, 0.38; $\mathrm{P}=0.33$, fixed effects model), with CWMT having higher ineffectiveness than AAT with CWMT. The heterogeneity was low $\left(\mathrm{P}=0.29, \mathrm{I}^{2}=13 \%\right)$ (Figure 6).

A meta-analysis based on a fixed-effects model was used to calculate the OR for the total effectiveness rate. The heterogeneity between the total effectiveness rates of AAT with CWMT and CWMT was analyzed, and the results showed an OR of 3.49 with $95 \%$ CI $(2.64,4.62)$. As indicated by the overall effect $\mathrm{P}$ value $<0.00001$ and $\mathrm{I}^{2}=13 \%$, the difference in total effectiveness rate between AAT with CWMT and CWMT was significant, with the AAT with CWMT group having a higher overall effectiveness rate 


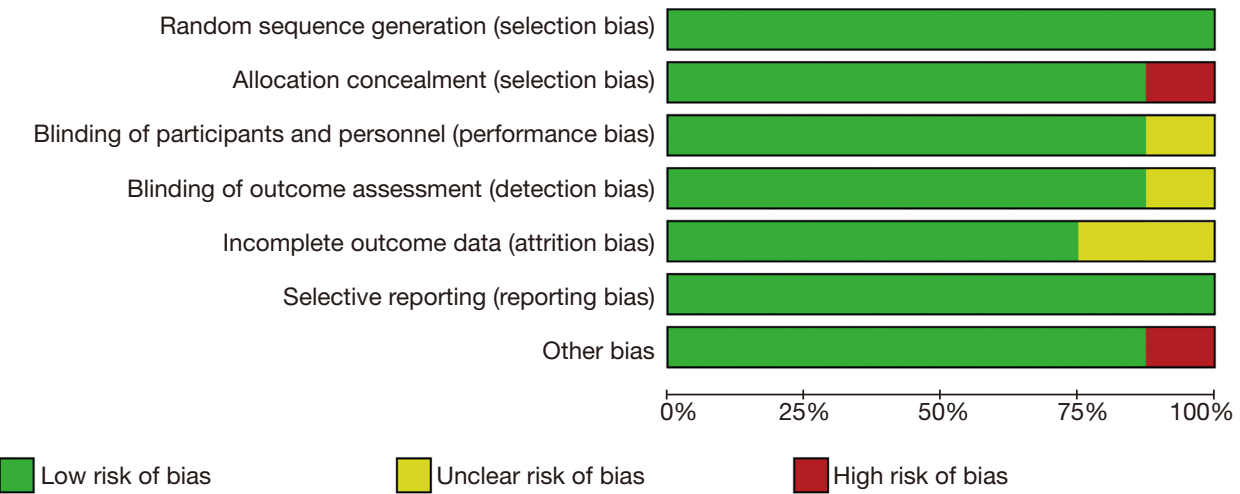

Figure 2 Risk of bias and applicability concerns graph for the included studies.

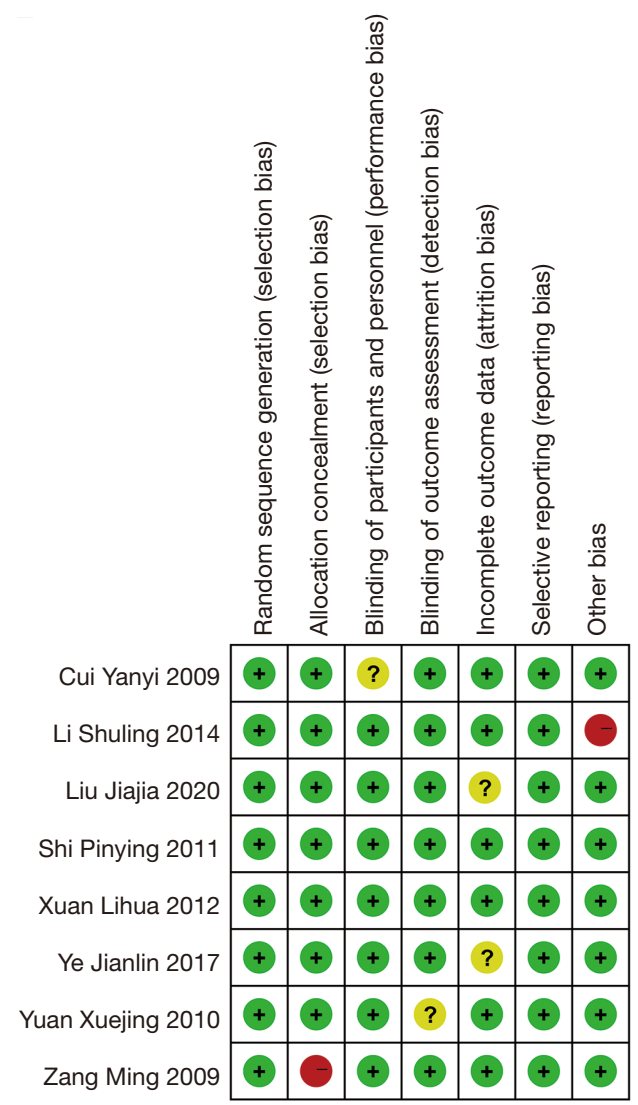

Figure 3 Risk of bias assessment results for each study. Green = low risk; yellow with question mark = unclear; and red = high risk.

than the CWMT group (Figure 7).

\section{Results of sensitivity and publication bias analyses}

All 8 studies reported the clinical effects of AAT with
CWMT and CWMT [Forest plots show that AAT with CWMT was less effective than CWMT, OR $=2.01,95 \%$ CI $(1.52,2.66), \mathrm{P}<0.0001, \mathrm{I}^{2}=36 \%$, Figure 4$]$. We performed a sensitivity analysis by removing the study of Liu et al. (17). The results showed only a slight change, with $\mathrm{I}^{2}$ rising from $36 \%$ to $38 \%$ (Figure 8 ), which indicated that the results of the included articles were robust.

A funnel plot was plotted to assess the publication bias of the studies in the meta-analysis. As can be seen in Figure 9, the plot is symmetrical in shape, indicating that there was no significant publication bias in this meta-analysis.

\section{Discussion}

In this meta-analysis, we found that AAT with CWMT had superior clinical and overall effects than CWMT in the treatment of bronchial asthma. CWMT also had higher ineffectiveness rate than AAT with CWMT. However, no difference in adverse events was found between AAT with CWMT and CWMT. These results were consistent with those of previous studies which found that AAT with CWMT can reduce the exacerbation severity and frequency, use of concomitant medications, and asthma degree. It has also been reported that prolonging the treatment course might enhance the efficacy of AAT with CWMT for patients with bronchial asthma (23).

AAT with CWMT and CWMT are two appropriate treatment modalities for bronchial asthma. CWMT is the primary clinical treatment for bronchial asthma (24), and the basic principle underlying this treatment is to achieve a long-term anti-inflammatory effect (25-28). Hormonal drugs can reduce inflammation induced by various factors, with inhaled glucocorticoids being the drug of choice for long-term treatment of asthma $(29,30)$. The 


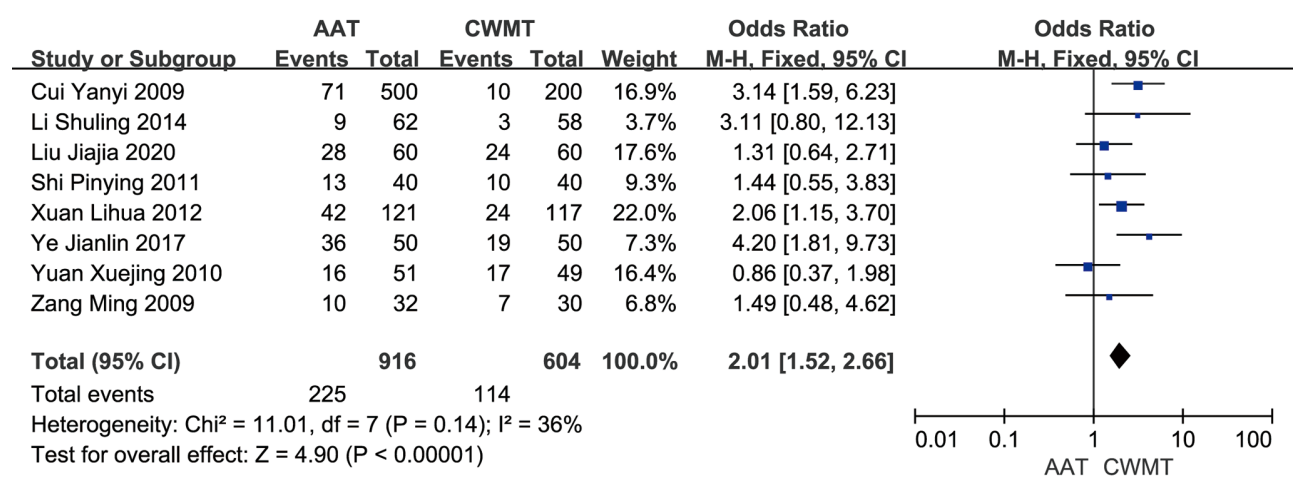

Figure 4 Comparison of the clinical effect of acupoint application with western medicine therapy (AAT with CWMT) with that of conventional western medicine (CWMT) in the treatment of bronchial asthma.

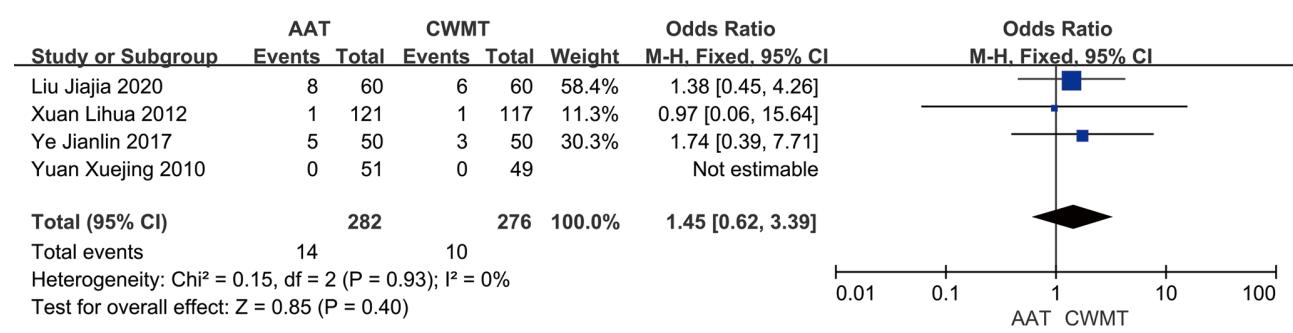

Figure 5 Forest plot comparing adverse events between acupoint application with western medicine therapy (AAT with CWMT) and conventional western medicine therapy (CWMT).

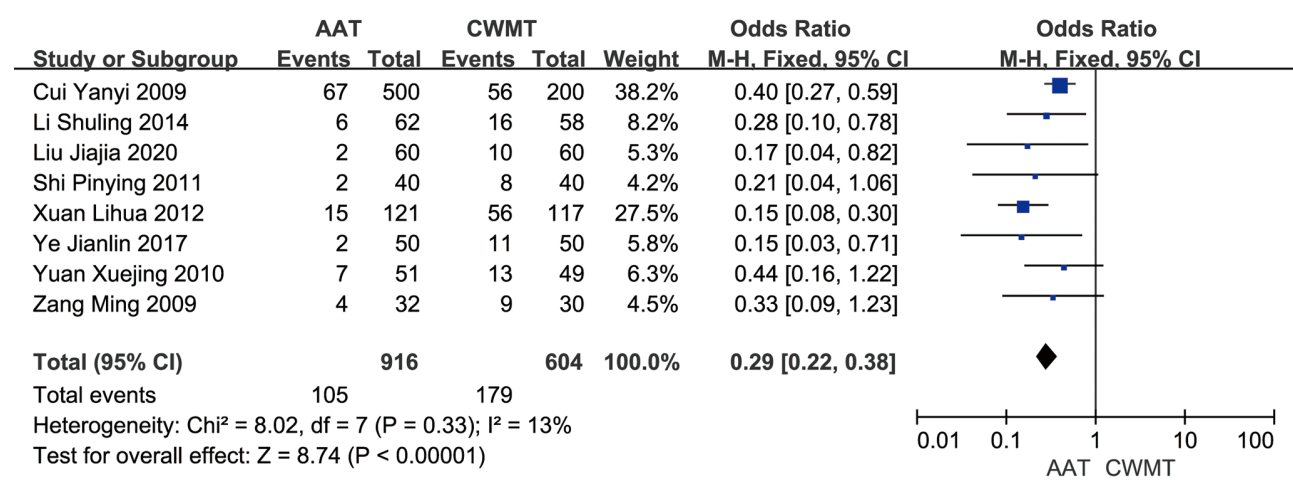

Figure 6 Forest plot comparing clinical outcomes between acupoint application with western medicine therapy (AAT with CWMT) and conventional western medicine therapy (CWMT): ineffectiveness rate.

drug's direct action on the airways is the characteristic of inhalation administration, which allows for high local drug concentration, precise efficacy, and more minor systemic adverse effects $(31,32)$. Receptor agonists can relax the bronchial smooth muscle, dilate the airways, and treat acute asthma attacks. However, acute episodes of bronchial asthma are characterized by dyspnea and hypoxemia, which require mechanical ventilation or oxygen therapy to maintain the patient's respiration and improve their symptoms. However, due to the lack of timely mechanical ventilation, some critically ill patients miss the optimal treatment time. 


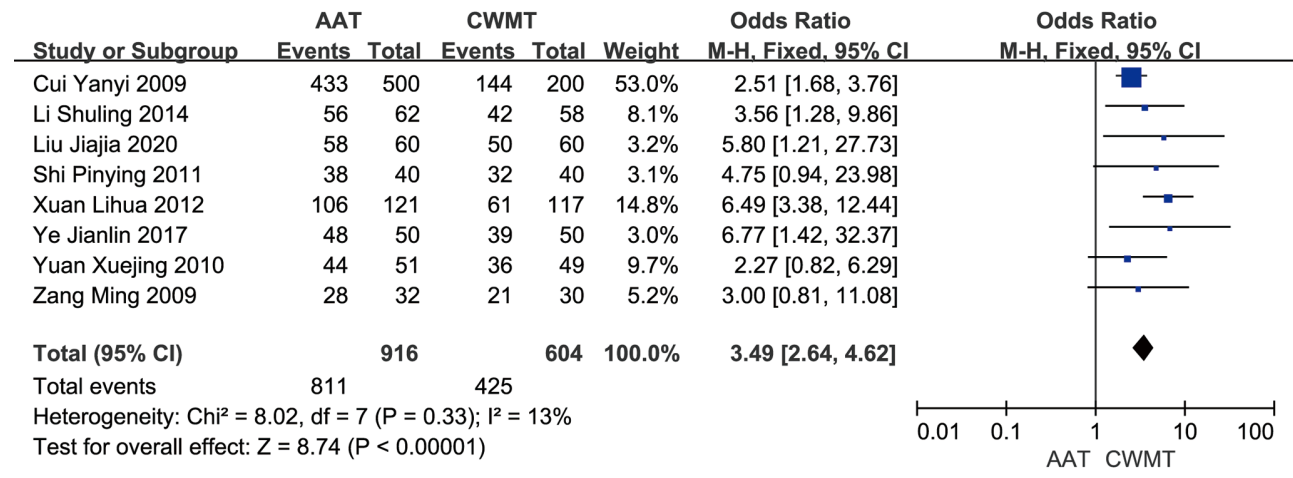

Figure 7 Forest plot comparing the overall effectiveness of acupoint application with western medicine therapy (AAT with CWMT) and conventional western medicine therapy (CWMT).

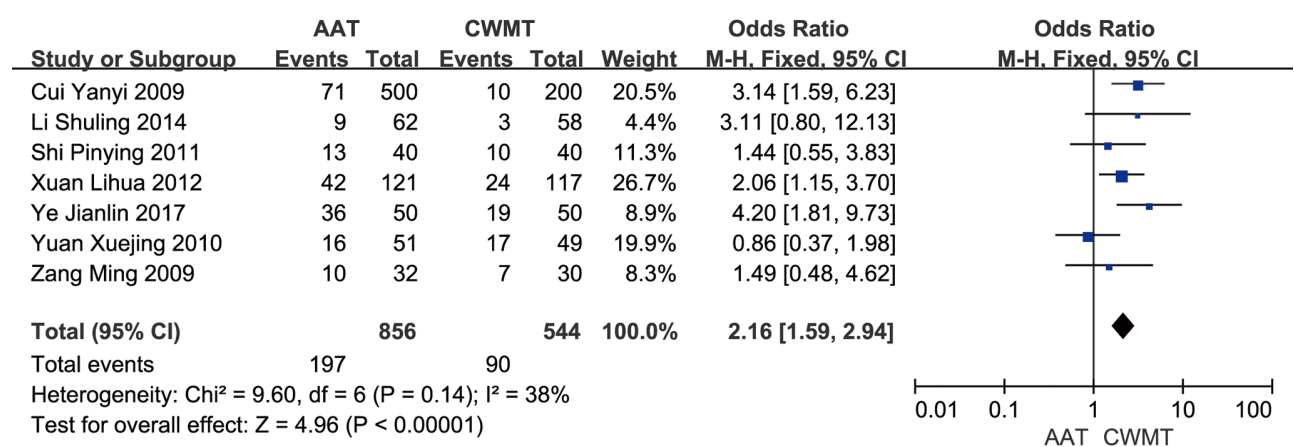

Figure 8 Sensitivity analysis results.

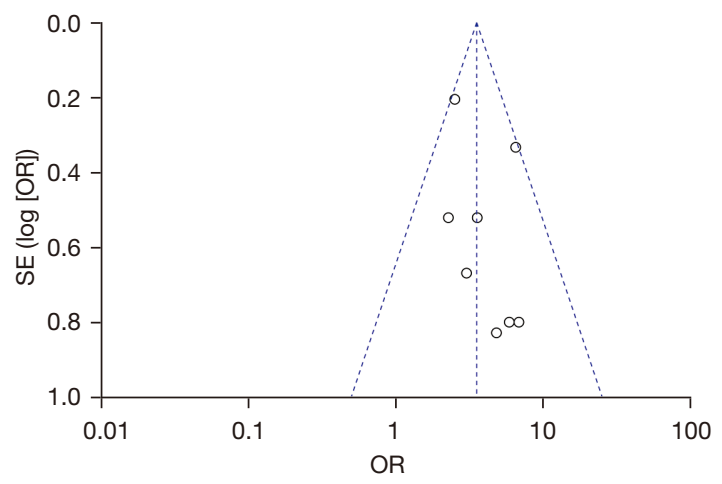

Figure 9 Funnel plot of estimated publication bias in the metaanalysis.

The duration of AAT is determined by the patient's skin reaction, and the patient's physical condition and tolerance should also be considered. Most of the western medicine used for bronchial asthma were phlegm-relieving, asthma-relieving, pungent, warming, and dispersing drugs $(33,34)$. Compared with CWMT, AAT with CWMT had the advantages including convenient operation and little adverse event. But, more evidence about AAT with CWMT is still imperative (34).

Bronchial asthma is a form of chronic nonspecific inflammation of the airway. The primary pathophysiology of bronchial asthma is the obstruction of airflow caused by bronchospasm and airway inflammatory swelling. Therefore, anti-inflammation and relieving bronchial smooth muscle spasms can effectively control asthma. The clinical observation results reported by Wu et al. (35) showed that AAT with CWMT is more effective than Western medicine alone in the treatment of bronchial asthma. Patients' serum total immunoglobulin $\mathrm{E}$ and interleukin-4 levels were significantly decreased, while the level of interferon gamma was considerably increased, which suggested that AAT with CWMT with CWMT can enhance Th1 cytokine function, inhibit Th2 cytokine function, and regulate the imbalance of Th1/Th2 cytokine ratio (34). TCM can control the lung, spleen, and kidney, strengthen the body, regulate immune function, and prevent 
bronchial asthma.

In conclusion, AAT with CWMT can significantly improve the curative rate in patients with asthma compared to CWMT, although there is no difference in adverse events between these two treatments. However, there were some limitations to our study, and there is still a need for studies from other regions and more detailed comparisons between AAT with CWMT and CWMT.

\section{Acknowledgments}

Funding: This study was supported by Jiangsu Science and Technology Department of China [BE2017771], National Natural Science Foundation of China [81674065] and Graduate student scientific research innovation projects in Jiangsu province [SJCX21_0779].

\section{Footnote}

Reporting Checklist: The authors have completed the PRISMA reporting checklist. Available at https://dx.doi. org/10.21037/apm-21-2507

Conflicts of Interest: All authors have completed the ICMJE uniform disclosure form (available at https://dx.doi. org/10.21037/apm-21-2507). The authors have no conflicts of interest to declare.

Ethical Statement: The authors are accountable for all aspects of the work in ensuring that questions related to the accuracy or integrity of any part of the work are appropriately investigated and resolved.

Open Access Statement: This is an Open Access article distributed in accordance with the Creative Commons Attribution-NonCommercial-NoDerivs 4.0 International License (CC BY-NC-ND 4.0), which permits the noncommercial replication and distribution of the article with the strict proviso that no changes or edits are made and the original work is properly cited (including links to both the formal publication through the relevant DOI and the license). See: https://creativecommons.org/licenses/by-nc-nd/4.0/.

\section{References}

1. Van Ganse E, Texier N, Dima AL, et al. Effects of shortand long-acting beta-agonists on asthma exacerbations: a prospective cohort. Ann Allergy Asthma Immunol
2020;124:254-60.

2. Xu P, Wang L, Chen D, et al. The application of proteomics in the diagnosis and treatment of bronchial asthma. Ann Transl Med 2020;8:132.

3. Kucuksezer UC, Ozdemir C, Cevhertas L, et al. Mechanisms of allergen-specific immunotherapy and allergen tolerance. Allergol Int 2020;69:549-60.

4. Jackson K, Bahna SL. Hypersensitivity and adverse reactions to biologics for asthma and allergic diseases. Expert Rev Clin Immunol 2020;16:311-9.

5. Wechsler ME, Ruddy MK, Pavord ID, et al. Efficacy and Safety of Itepekimab in Patients with Moderate-to-Severe Asthma. N Engl J Med 2021;385:1656-68.

6. Nwaru BI, Shah SA, Tibble H, et al. Hormone Replacement Therapy and Risk of Severe Asthma Exacerbation in Perimenopausal and Postmenopausal Women: 17-Year National Cohort Study. J Allergy Clin Immunol Pract 2021;9:2751-2760.e1.

7. Nejatbakhsh Samimi L, Fallahpour M, Khoshmirsafa M, et al. The impact of $17 \beta$-estradiol and progesterone therapy on peripheral blood mononuclear cells of asthmatic patients. Mol Biol Rep 2021;48:297-306.

8. Wang J, Zhai C, Wang Q, et al. Determinants of ICS therapy adherence in patients with asthma. Am J Manag Care 2021;27:e36-41.

9. Gans MD, Gavrilova T. Understanding the immunology of asthma: Pathophysiology, biomarkers, and treatments for asthma endotypes. Paediatr Respir Rev 2020;36:118-27.

10. Chan CW, Lee SC, Lo KC, et al. Tian jiu therapy for the treatment of asthma in adult patients: a meta-analysis. J Altern Complement Med 2015;21:200-7.

11. Su L, Meng L, Chen R, et al. Acupoint Application for Asthma Therapy in Adults: A Systematic Review and Meta-Analysis of Randomized Controlled Trials. Forsch Komplementmed 2016;23:16-21.

12. Lai $X, \operatorname{Li} Y$, Fan $Z$, et al. An analysis of therapeutic effect of drug acupoint application in 209 cases of allergic asthma. J Tradit Chin Med 2001;21:122-6.

13. Chen WH, Xin K, Cai CA, et al. Observation on therapeutic effect of acupoint application of Chinese medicine on bronchial asthma. Zhongguo Zhen Jiu 2009;29:272-4.

14. Zhao SM, Wang HS, Zhang C, et al. Repeated Herbal Acupoint Sticking Relieved the Recurrence of Allergic Asthma by Regulating the Th1/Th2 Cell Balance in the Peripheral Blood. Biomed Res Int 2020;2020:1879640.

15. Xuan L, Yu BY, Xu F, et al. Clinical study on the 
prevention and treatment of bronchial asthma by acupoint application with different stimulation intensity. CJTCMP 2012;7:1807-10.

16. Li S, Yang LH, Ma C. Effect of Acupoint Application of traditional Chinese medicine on senile cough variant asthma in dog days. Chin J Geron 2014;24:7103-4.

17. Liu J. Observation on therapeutic effect of Acupoint Application on bronchial asthma. Shanghai Journal of Acupuncture and Moxibustion 2020;12:1541-5.

18. Zang M, Xuan LH, Feng SJ. Preventive treatment Observation on the therapeutic effect of acupoint sticking on bronchial asthma. Chin Arc Tra Chin Med 2009;12:2619-21.

19. Cui Y. Five hundred cases of asthma in remission stage treated with Acupoint Application of Jiexin ointment and Sanfu Tianxin. J Tra Chin Med 2009;5:438-9.

20. Shi P, Zhu JF, You JS, et al. Clinical observation on Acupoint Application of Wu's foam ointment combined with Seretide inhalation in the prevention and treatment of 40 cases of childhood asthma. J Tra Chin Med 2011;4:314-6.

21. Ye J, Huang JL, Luo XY. Clinical observation on moxibustion combined with Acupoint Application in the treatment of children with asthmatic bronchitis. Hebei J TCM 2017;10:1559-61.

22. Yuan X, Sun YQ, Wang SM, et al. Clinical study of Guben Fangxiao decoction combined with Acupoint Application in the treatment of 100 children with asthma in remission stage. CJTCMP 2010;12:2306-9.

23. Ll BL, Wang YL, Yang Q, et al. Effect of Acupoint Application Therapy of Summer Treatment for Winter Diseases on Nerve-Endocrine-Immune Network System in Patient with Non-Acute Attack Asthma. Zhongguo Zhong Xi Yi Jie He Za Zhi 2017;37:68-71.

24. Czock D, Keller F, Rasche FM, et al. Pharmacokinetics and pharmacodynamics of systemically administered glucocorticoids. Clin Pharmacokinet 2005;44:61-98.

25. Barnes PJ. Glucocorticosteroids. Handb Exp Pharmacol

Cite this article as: $\mathrm{Hu} \mathrm{J}$, Zhang $\mathrm{C}$, Zhao S, Ge K, Di K, Wang H, Liu L. A systematic review and meta-analysis of acupoint application combined with western medicine therapy in the treatment of bronchial asthma. Ann Palliat Med 2021;10(11):1147311481. doi: 10.21037/apm-21-2507
2017;237:93-115.

26. Rodriguez JM, Monsalves-Alvarez M, Henriquez S, et al. Glucocorticoid resistance in chronic diseases. Steroids 2016;115:182-92.

27. Ora J, Calzetta L, Matera MG, et al. Advances with glucocorticoids in the treatment of asthma: state of the art. Expert Opin Pharmacother 2020;21:2305-16.

28. Iikura M, Hojo M, Sugiyama H. Glucocorticoids and mepolizumab in eosinophilic asthma. $\mathrm{N}$ Engl J Med 2014;371:2433-4.

29. Jackson DJ, Bacharier LB, Mauger DT, et al. Quintupling Inhaled Glucocorticoids to Prevent Childhood Asthma Exacerbations. N Engl J Med 2018;378:891-901.

30. He Y, Shi J, Nguyen QT, et al. Development of highly potent glucocorticoids for steroid-resistant severe asthma. Proc Natl Acad Sci U S A 2019;116:6932-7.

31. Agarwal R, Aggarwal AN, Dhooria S, et al. A randomised trial of glucocorticoids in acute-stage allergic bronchopulmonary aspergillosis complicating asthma. Eur Respir J 2016;47:490-8.

32. Alangari AA. Genomic and non-genomic actions of glucocorticoids in asthma. Ann Thorac Med 2010;5:133-9.

33. Wen BL, Liu BY, Jin P, et al. Clinical research of acupoint application for "treatment of winter disease in summer" used to prevent and treat bronchial asthma in children. J Tradit Chin Med 2012;32:31-9.

34. Yang XC, Yin T, Gao Q, et al. The immunomodulatory effect of acupoint application for childhood asthma: a systematic review and meta-analysis. Evid Based Complement Alternat Med 2015;2015:896247.

35. Wu X, Peng J, Li G, et al. Efficacy evaluation of summer acupoint application treatment on asthma patients: a two-year follow-up clinical study. J Tradit Chin Med 2015;35:21-7.

(English Language Editor: J. Reynolds) 